\title{
PERTURBATION FONCTIONNELLE PROLONGEE DE LA SECRETION DE PROLACTINE APRES CHIRURGIE SOUS NEUROLEPTANALGESIE
}

\author{
Yves Jullien, Gérard Desch, Andrée Bonardet, Dominique Alloua, \\ Marc deRodez, Bernard Descomps et JacQues du Callar
}

\begin{abstract}
Le taux de base de prolactine ainsi que la réponse de l'hormone au test de stimulation par l'halopéridol sont mesurés chez neuf femmes avant et après une intervention réglée de chirurgie gynécologique courante réalisée sous neuroleptanalgésie de type dextromoramide-dropéridol.

La veille de l'intervention, une augmentation de prolactine de $5,4 \pm 1,9 \mathrm{ng} / \mathrm{ml}$ est observée après $0.25 \mathrm{mg}$ d'halopéridol I.M. alors que le lendemain de l'opération, la réponse à la même stimulation est abolie $(p<0,025)$.

Le surlendemain de l'intervention, bien qu'une dose dix fois plus importante d'halopéridol soit administrée (2,5 mg I.M.) la réponse de prolactine n'est que du même ordre de grandeur que celle observée en pré-opératoire $(5,8 \pm 1,2 \mathrm{ng} / \mathrm{ml}:$ N.S.)

Ces résultats liés aux faibles taux de bases : $1,9 \pm 0,6$ et $1,1 \pm 0,3 \mathrm{ng} / \mathrm{ml}$ observés 24 et 48 heures après l'opération (contre $6,2 \pm 1,4 \mathrm{ng} / \mathrm{ml}$ la veille de l'intervention, $\mathrm{p}<0,005$ dans les deux cas) mettent en évidence une perturbation fonctionnelle prolongée de la sécrétion de prolactine.
\end{abstract} Mots CLeFs: Prolactine - Protection - Hypophyse - Neuropletanalgésie - Neuro-
leptiques - Système dopaminergique.

LA LIBÉRATION de prolactine (P.R.L.) par l'adénohypophyse au cours du stress, est un fait bien établi. ${ }^{9,14}$ Cependant, l'étude peropératoire de la sécrétion de P.R.L. montre que l'intensité de l'agression chirurgicale a moins d'importance que I'anesthésie elle-même. ${ }^{1}$ En particulier, la sécrétion de P.R.L. est fortement stimulée par les morphiniques ${ }^{12,15}$ et les neuroleptiques. ${ }^{5}$ En conséquence, la P.R.L. n'a qu'un faible intérêt comme indicateur de l'intensité du stress opératoire. ${ }^{1}$

Les travaux étudiant l'évolution post-opératoire de la P.R.L. sont par ailleurs peu nombreux. ${ }^{4,9}$ L'objectif du présent travail est d'étudier-dans des conditions standardisées- l'évolution post-opératoire de la P.R.L. ainsi que sa réponse au test fonctionnel de stimulation par l'halopéridol. L'élévation de la P.R.L. consécutive à une dose déterminée d'halopéridol est constante pour un individu donné, la réponse maximale survenant dans les 90 à 120 minutes

Yves Jullien, Dominique Alloua, Marc de Rodez et Jacques du Cailar, Departement d'AnesthésieRéanimation A, Centre Hospitalier Universitaire, Hópital Saint-Eloi; Gérard Desch, Andrée Bonardet et Bernard Descomps, Laboratoire de Biochimie C, Centre Hospitalier Universitaire, Hôpital SaintCharles; 34059 Montpellier Cedex, France. suivant l'injection. Il existe d'autre part une corrélation linéaire étroite entre cette réponse et la dose d'halopéridol dans l'intervalle de 0.25 à $1,25 \mathrm{mg}^{5}$ De ce fait, le test a l'halopéridol semble être un moyen adéquat d'évaluer la réactivité fonctionnelle de l'hypophyse en postoperatoire.

\section{Materiel et Methode}

Le présent travail porte sur neuf patientes bénéficiant d'une intervention réglée de chirurgie gynécologique courante. Le type d'intervention est indique dans le tableau I. L'âge moyen de ces femmes est de $47 \pm 8$ ans, leur poids est de $59 \pm 9 \mathrm{~kg}$ et leur taille de $159,8 \pm 8 \mathrm{~cm}$. Aucune d'elle ne présente de tare organique et plus spécialement d'anomalie endocrinienne. Aucune ne bénéficie d'un traitement médical ni même de l'administration d'oestro-progestatifs.

L'étude a été conduite conformément à la déclaration d'Helsinki et le consentement écrit des patientes a été obtenu.

\section{Protocole anesthésique}

La prémédication associe diazépam $20 \mathrm{mg}$ atropine $0,5 \mathrm{mg}$ intramusculaire une heure avant l'anesthésie.

Can. Anaesth. Soc. J., vol. 29, no. 5, September 1982 


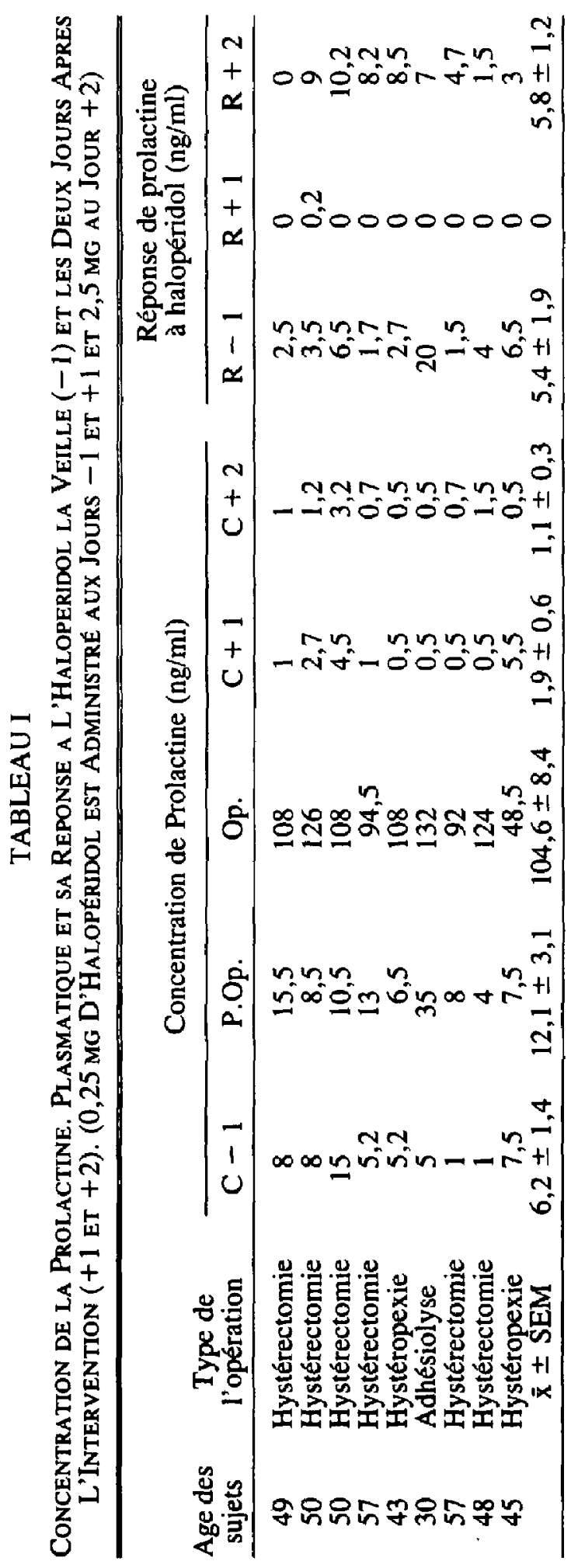


L'induction est effectuée par injection intraveineuse de $5 \pm 0,47 \mathrm{mg}$ de dextromoramide (morphinomimétique puissant apparenté à la méthodone) et de 23,33 $\pm 3,54 \mathrm{mg}$ de dropéridol.

La narcose est obtenue par l'injection de thiopentone $283 \pm 130 \mathrm{mg} \mathrm{I.V.}$

La respiration est contrôlée (volume courant $10 \mathrm{ml} \cdot \mathrm{kg}^{-1}$ fréquence 12) au moyen d'un mélange protoxyde d'azote oxygène à $50 \mathrm{p} .100$.

L'intubation trachéale est précédée de l'injection de succinylcholine $50 \mathrm{mg}$ (dose standard).

La myorésolution per-opératoire est obtenue au moyen de bromure de pancuronium 5,22 \pm $0,83 \mathrm{mg}$.

L'entretien de l'anesthésie comporte l'injection de dextromoramide $5 \pm 2,2 \mathrm{mg}$, thiopentone $181 \pm 90 \mathrm{mg}$ et bromure de pancuronium $1,33 \pm 0,75 \mathrm{mg}$.

\section{Protocole analytique}

Les prélèvements de sang veineux périphérique sont effectués de la manière suivante :

1 - La veille de l'intervention $\left(\mathrm{J}_{-1}\right)$ entre $17 \mathrm{et}$ 20 heures, il est d'abord prélevé deux échantillons - à un quart d'heure d'intervalle - dont la moyenne des concentrations en P.R.L. détermine la concentration basale à $J_{-1}$ identifiée : C-1. Il est ensuite injecté $0,25 \mathrm{mg}$ d'halopéridol intramusculaire. Trois prélèvements sont alors effectués d $90-105$ et 120 minutes de l'injection. Parmi ces trois prélèvements, la concentration en P.R.L. la plus élevée servira à calculer par différence avec le taux de base, la réponse à $J_{-1}$ identifiée : $\mathbf{R}-\mathbf{1}$.

2 - Le jour de l'intervention deux prélèvements sont effectués l'un lors de l'arrivée de la patiente sur la table d'opération à $8 \mathrm{~h}$ (p.op), l'autre en per-opératoire (op) une heure après l'incision. C'est volontairement qu'il n'a pas été effectué deux prélèvements de référence, les opérées n'étant pas considérées à ce moment comme en état basal. Le but de ces deux prélèvements est simplement d'objectiver le pic per-operatoire de P.R.L. sans prétendre en identifier la cause principale.

3 - Le lendemain de l'intervention $\left(\mathrm{J}_{+1}\right):$ les mêmes cinq prélèvements que le jour préoperatoire sont effectuées avec la même injection de $0,25 \mathrm{mg}$ d'haloperidol, sensiblement au même moment du nycthémère. Ces prélèvements permettent de définir la concentration basale à $\mathrm{J}_{+1}: \mathrm{C}+1$ ainsi que la réponse de la P.R.L. : R + 1 .
4-Le surlendemain de l'intervention $\left(\mathrm{J}_{+2}\right)$, la même manipulation est répétée mais avec injection de $2,5 \mathrm{mg}$ d'halopéridol ce qui permet de calculer $C+2$ et $R+2$.

Tous les échantillons sanguins sont centrifugés dans les minutes qui suivent leur recueil et le plasma prélevé est immédiatement congelé. Le dosage de P.R.L. est effectué par la méthode radio-immunologique de l'association C.E.A., I.R.E., S.O.R.I.N. sans modification*. ${ }^{10}$ Chaque échantillon fait l'objet d'un triple dosage.

Le coefficient de variation inter-essais évalué sur un pool de plasma provenant de femmes normales est de 7,5 p. 100 (15 déterminations) et le coefficient de variation intra-essai déterminé dans les mêmes conditions de 5,9 p. 100. La limite inférieure de sensibilité est de $0,68 \mathrm{ng} \cdot \mathrm{ml}^{-1}$ et les taux plasmatiques normaux de P.R.L. chez la femme sont de 3 à $13 \mathrm{ng} \cdot \mathrm{ml}^{-1}$.

Les comparaisons statistiques sont effectuées au moyen du test de Student apparié ; sauf spécification contraire, tous les résultats sont exprimés en moyenne \pm écart standard à la moyenne $(\bar{x} \pm$ SEM $)$.

\section{Resultats}

L'évolution des taux plasmatiques de P.R.L. entre $J_{-1}$ et $J_{+2}$ est représentée par la figure 1 ; les valeurs individuelles et les moyennes sont indiquées dans le tableau I.

Il existe une très importante diminution des taux de base de P.R.L. les deux jours suivants l'intervention ( $\mathrm{p}<0,005$ dans les deux cas).

Au cours de l'intervention, le taux plasmatique de P.R.L. s'élève brutalement à huit fois la valeur pré-opératoire et dix-sept fois le taux basal de la veille ( $p<0,001$ dans les deux cas).

La réponse moyenne de P.R.L. lors de la stimulation de l'halopéridol est représentée dans la figure 2, les valeurs individuelles ainsi que les moyennes sont indiquées dans le tableau I.

Le lendemain de l'intervention, la réponse de la P.R.L. à $0,25 \mathrm{mg}$ d'halopéridol est quasiment supprimée $(p<0,025)$ et un seul sujet se montre capable de répondre à cette stimulation.

Le surlendemain de l'intervention, bien qu'une dose d'halopéridol dix fois supérieure soit administrée $(2,5 \mathrm{mg})$ la réponse de P.R.L. n'est que du même ordre de grandeur qu'à $J_{-1}$ (N.S.).

*P.R.O.L. - R.I.A. 200 foumi par C.E.A. - I.R.E. Gif sur Yvette - France. 


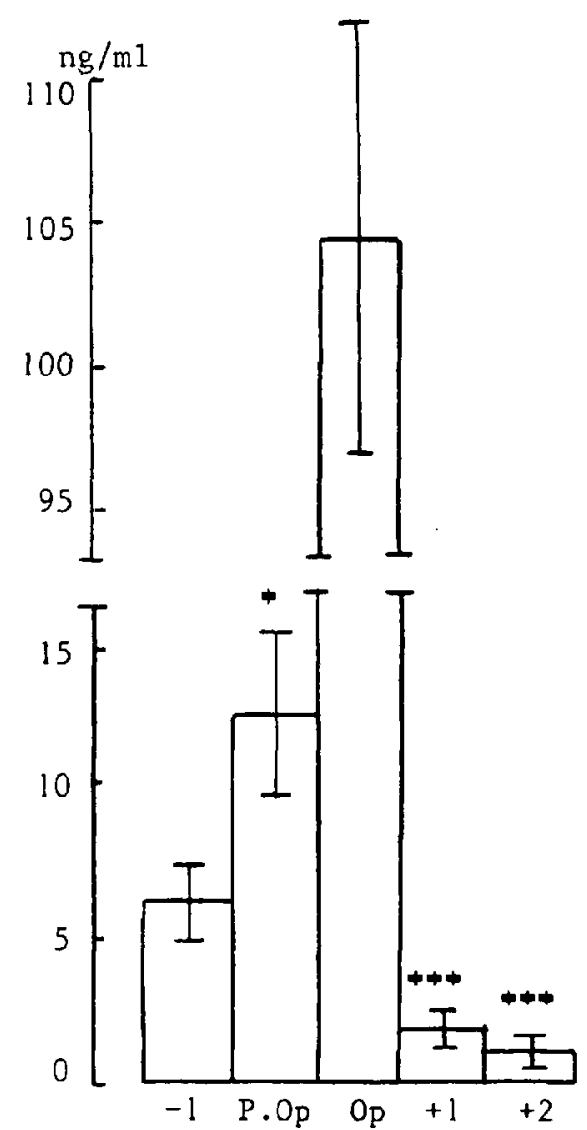

FIGURE 1 Concentrations basales de prolactine. --1 : Veille de l'intervention

$-+1,+2$ : Lendemain et surlendemain de l'intervention

-P. Op. : immédiatement après l'induction

- Op. : $1 \mathrm{~h}$ après l'incision

Valeurs exprimées : $\overline{\mathrm{x}} \pm$ SEM de neuf sujets. Significativité:

$+: \mathrm{p}<0,05$ $+++: \mathrm{p}<0,005$

$++++: p<0,001)$

\section{Comparaisons avec les} valeurs a $\mathbf{J}_{-1}$.

\section{Discussion}

Le taux de P.R.L. pré-opératoire est supérieur au taux de base de $\mathrm{J}_{-1}$. Cette différence est vraisemblablement due à la fois à l'agression émotionnelle et aux variations nycthémérales de la P.R.L. En effet, le prélèvement per-opératoire est effectué à $8 \mathrm{~h}$, heure à laquelle le pic nocturne de P.R.L. peut ne pas avoir totalement disparu. 11 $\mathrm{ng} / \mathrm{ml}$

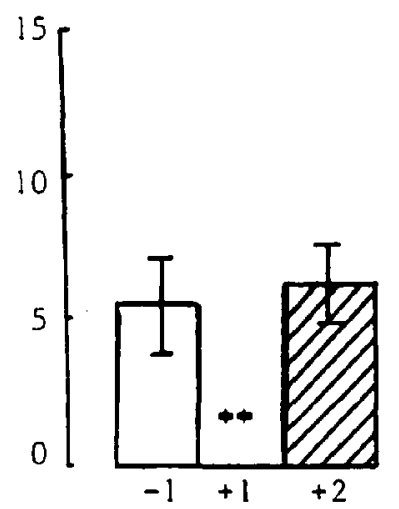

FIGURE 2 Augmentation de prolactine après halopéridol.

-1 : Veille de l'intervention

$-+1,+2$ : Lendemain et surlendemain de l'intervention

$-0,25 \mathrm{mg}$ d'halopéridol est injecté à $\mathrm{J}_{-1}$ et $\mathrm{J}_{+1}$ et $2,5 \mathrm{mg}$ à $\mathrm{J}_{+2}$ (hachures)

Valeurs exprimées : $\bar{x} \pm$ SEM de neuf sujets. Significativité:

$++:<p<0,025$ Comparaisons avec les valeurs à $J_{-1}$.

La très importante libération de P.R.L. peropératoire peut être rattachée à trois facteurs : d'une part la stimulation hypophysaire par de fortes doses de morphinomimétiques, ${ }^{12,15}$ d'autre part la suppression du tonus dopaminergique inhibiteur par le neuroleptique ${ }^{5}$ et enfin l'agression chirurgicale elle-même. ${ }^{9,14}$ La part respective de chacun de ces facteurs ne peut être évaluée au terme du présent travail; elle reste à déterminer. La diminution des taux de base à $\mathbf{J}_{+1}$ et $\mathbf{J}_{+2}$ confirme la dépression prolongée de P.R.L. après chirurgie sous neuroleptanalgésie de type dextromoramidedropéridol. ${ }^{4}$ Les faibles réponses à l'halopéridol démontrent une importante perturbation fonctionnelle de la sécrétion de prolactine.

Cette variation ne peut pas être attribuée à un épuisement hypophysaire contemporain de l'acte chirurgical. En effet, les contenus hypophysaires en prolactine de l'être humain est de 100 a $300 \mu \mathrm{g}^{3}$ alors que la clairance métabolique de l'hormone est de l'ordre de $1000 \mu \mathrm{g} /$ jour. ${ }^{13}$

Le contenu hypophysaire en prolactine se renouvelle donc de trois à dix fois par 24 heures. De plus, des taux mesurables de prolactine circulante persistent à $\mathrm{J}_{+1}$ et $\mathrm{J}_{+2}$. Enfin, il existe 
une réponse mesurable de à $\mathbf{J}_{+2}$, alors que les taux de base sont à leur niveau minimum.

Ainsi, une inhibition de la sécrétion hypophysaire est plus vraisemblablement responsable des résultats observés qu un épuisement de son contenu.

Une telle inhibition peut s'expliquer:

- soit comme le résultat d'un rétro-contrôle négatif conséquence des taux extrêment élevés de prolactine réalisés en per-opératoire,

- soit par une augmentation du tonus dopaminergique liée à un effet présynaptique prolongé du dropéridol, ${ }^{2,8}$

- soit enfin, par une association de ces deux mécanismes.

Quelle que soit la mise en jeu, l'anesthésie de type dextromoramide dropéridol entraîne une dépression prolongée de prolactine liée à une perturbation fonctionnelle de sa régulation. Les conséquences physiologiques, si elles existent, restent à déterminer.

\section{BIBLIOGRAPHIE}

1. Bellman, O. \& Stoeckel, H. The influence of anaesthesia on prolactin secretion in man. In : Stoeckel, H., Oyama, T., Endocrinology in anaesthesia and surgery. Berlin, Heidelberg, New-York, Springer Verlag, 1980, 1 vol., 203 p. (pp. 101-111).

2. Biggio, G., Casu, M., Klimer, V \& Gessa, G.L. Dopamine synthesis : tolerance to haloperidol and supersensitivity to apomorphine depend on presynaptic receptors. In : CATABENI, Long Term effects of neuroleptics (Adv. Biochim. Physcho-pharmacol.), New-York, Raven Press, 1980, vol. 24, 1243 p. (pp. 17-22).

3. Hwang, P., Friesen, H., Hardy, J. \& WilanSKY, D. Biosynthesis of human growth hormone and prolactin by normal pituitary glands and pituitary adenomas. J. Clin. Endocrinol. Metab. 33: 1 (1971).

4. Jullien, Y., de Rodez, M., Bonardet, A. Desch, G., Descomps, B. et du Cailar, J Comparaisons des taux sanguins de prolactine et de somatotrophine en post-opératoire après deux modes d'anesthésie. Ann. Anesth. Franç. 21: 459 (1980).
5. Langer, G., Sachar, E.J., Halpern, F.S., GRUEN, P.H. \& SALMON, M. The prolactin response to neuroleptic drugs. A test of dopaminergic blockade : neuroendocrine studies in normal man. J. Clin. Endocrinol. Metab. 35 : 996 (1977).

6. Melmed, S., Carlson, H.E., Brigcs, J. \& Hershman, J.M. Autofeedback of prolactin in cultured prolactin-secreting pituitary cells. Hormone Res. 12: 340 (1980).

7. Moore, K.E., Desmaret, K.T. \& Johnston, C.A. Influence of prolactin on dopaminergic neurolal systems in the hypothalamas. Federation Proc. $39: 2912$ (1980).

8. Muller, P., Syensson, T.H. \& Carlsson, A. Pre and postsynaptic mechanism in haloperidol induced sensitization to dopaminergic agonists. In : CATTABENI, Long term effects of neuroleptics (Adv. Biochem. Psychopharmacol. NewYork Raven Press, 1980, vol. 24, 1243 p. (pp. 69-74).

9. NoEl, G.L., SuH, H.K., Stone, J.G. \& FRANTZ, A.G. Human prolactin and growth hormone release during surgery and other conditions of stress. J. Clin. Endocrinol. Metab. 35: 840 (1972).

10. Rueter, A.M., Kennes, F., Gevaert, Y, \& FRANCHIMONT, $P$. Dosage radio-immunologique homologue de la prolactine humaine. Path. Biol. (Paris) 23: 761 (1975)

11. Sassin, J.F., Frantz, A.G., Weitzman, E.D. \& KAPEN, S. Human prolactin : 24 hours pattern with increased during sleep. Science 17; 1205 (1972).

12. Shata, C.J., Frederickson, R.C.A., DininGER, N.B. \& J JCKSON, L. Enkephalin analogues and naloxone modulate the release of growth hormone and prolactin : evidence for regulation by an endogenous opioid peptide in brain. Life Science 21: 853 (1977).

13. Sievertsen, G.D., LIM, V.S., Nakawatase, C. \& Frohman, L.A. Metabolic clearance and secretion rates of human prolactin in normal subjects and in patients with chronic renal failure. J. Clin. Endocrinol. Metab. 50: 846 (1980).

14. SOWERs, J.R., RAJ, R.P. HERSHMAN, J.M Carlson, H.E. \& MacCallum, R.W. The effect of stressful diagnostic studies and surgery on anterior pituitary hormone release in man. Acta Endocrinol. (Kbh) 86: 25 (1977).

15. Tolis, G., Hickey, J. \& Guyada, $H$. Effect of morphine on serum G.H., P.R.L., T.S.H. and cortisol in man. J. Clin. Endocrinol. Metab. 4I: 797 (1975).

\section{ABSTRACT}

Plasma level of prolactin and its response to the injection of haloperidol were measured in nine women before and after elective gynecological surgery under neuroleptanalgesia using dextromoramidedroperidol combination.

The day before the operation, the injection of haloperidol $0.25 \mathrm{mg}$ intramusculary increases the concentration of PRL by $5.4 \pm 1.9 \mathrm{ng} / \mathrm{ml}$, while this response is completely abolished the day following the operation.

Two days later, a tenfold increase in the dose of haloperidol ( $2.5 \mathrm{mg}$ I.M.) is necessary to obtain a PRL response that equals that observed pre-operatively $(5.8 \pm 1.2 \mathrm{ng} / \mathrm{ml})$.

The low plasma levels observed 24 and 48 hours post-operatively compared to the pre-operative level, illustrate a prolonged functional disturbance of the secretion of PRL. 